4-1-1992

\title{
Scaling Dynamics of Aerosol Coagulation
}

B. J. Olivier

C. M. Sorensen

Thomas W. Taylor

Cleveland State University, t.taylor@csuohio.edu

Follow this and additional works at: https://engagedscholarship.csuohio.edu/sciphysics_facpub

Part of the Physics Commons

How does access to this work benefit you? Let us know!

Publisher's Statement

Copyright 1992 American Physical Society. Available on publisher's site at http://pra.aps.org/ abstract/PRA/v45/i8/p5614_1.

Original Citation

Olivier, B. J. , C. M. Sorensen, and Thomas W. Taylor. "Scaling Dynamics of Aerosol Coagulation." Physical Review A 45 (1992): 5614-5623.

\section{Repository Citation}

Olivier, B. J.; Sorensen, C. M.; and Taylor, Thomas W., "Scaling Dynamics of Aerosol Coagulation" (1992). Physics Faculty Publications. 3.

https://engagedscholarship.csuohio.edu/sciphysics_facpub/3

This Article is brought to you for free and open access by the Physics Department at EngagedScholarship@CSU. It has been accepted for inclusion in Physics Faculty Publications by an authorized administrator of EngagedScholarship@CSU. For more information, please contact library.es@csuohio.edu. 


\title{
Scaling dynamics of aerosol coagulation
}

\author{
B. J. Olivier* \\ Sandia National Laboratories, Albuquerque, New Mexico 87185 \\ and Department of Physics, Kansas State University, Manhattan, Kansas 66506 \\ C. M. Sorensen \\ Department of Physics, Kansas State University, Manhattan, Kansas 66506 \\ T. W. Taylor \\ Department of Physics, Cleveland State University, Cleveland, Ohio 44115
}

(Received 18 December 1991)

\begin{abstract}
A combination of static and quasielastic light scattering and the theory of scaling solutions to Smoluchowski's equation was used to determine the absolute coagulation rate $K_{0}^{\prime}$ and kernel homogeneity $\lambda$ of a coagulating liquid-drop aerosol. Droplet sizes ranged from 0.23 to $0.42 \mu \mathrm{m}$, implying Knudsen numbers in the range 0.26 and 0.14 . The temporal evolution of the number concentration $M_{0}$ and the modal radius $r_{M}$ of an assumed zeroth-order log-normal distribution showed near-power-law behavior similar to that predicted by the scaling theory. From the temporal scaling behavior of $M_{0}(t)$ and $r_{M}(t)$, the absolute coagulation rate was calculated. The coagulation rates from each method were in good agreement. The rate also agreed well with theory that corrected the Brownian rate, good for the continuum regime, by the average Cunningham correction factor. In addition, the time dependence of the moments $M_{0}$ and $r_{M}$, hence the determination of $K_{0}^{\prime}$, was in good agreement with a real-time numerical solution of Smoluchowski's equation for initial conditions analogous to our experimental ones.
\end{abstract}

PACS number(s): 82.70.Rr, 82.70.Kj, 82.20.-w

\section{INTRODUCTION}

The past decade has witnessed a renaissance of interest in fundamental coagulation phenomena. Our ability to describe and quantify random aggregates as fractal $[1,2]$ as well as the further developments, since the pioneering work of Friedlander and Lushnikov [3-6], of dynamic scaling concepts used to describe the real-time evolution of particle-size distributions [7] has largely accounted for the revival. This enhanced interest has led to a deeper and more general understanding of the dynamic nature of coagulation processes.

The majority of recent experimental coagulation studies have involved the colloidal phase of matter. These studies [8-12] have used quasielastic and static lightscattering techniques as experimental probes and primarily concerned themselves with cluster morphologies and relative growth rates. For the most part, the studies were directed towards characterizing scaling properties such as (1) the static, length-scale invariant nature of the aggregate via the fractal dimension $D_{f}$ and (2) the dynamic scaling nature of the aggregate-size distribution. Additional studies $[13,14]$ have further identified the exponential shape of the large-radius end of some of these scaling distributions. No effort, however, has been directed toward applying recent scaling concepts to measure absolute quantities such as coagulation-rate constants for particulate systems.

Particulate systems can be characterized by a Knudsen number $\mathrm{Kn}$ which is defined as the ratio of the mean free path of the solvent molecules $l$ to the particle radius $r$.
For colloidal systems, one has $\mathrm{Kn} \approx 0$. These relatively dense systems generally harbor complex particle-particle and particle-solvent interactions which are not well understood. The coagulation process in these systems is usually initiated by the addition of a suitable coagulant so to reduce stabilizing interparticle forces. The coagulant, however, further complicates particle-particle interactions within the colloid and the role of the coagulant during the coagulation process is also poorly understood.

Gas-phase systems, or aerosols, represent another type of particulate system of great importance and common occurrence. Less effort, however, has been directed toward understanding coagulation processes inherent in aerosols using the recently advanced scaling concepts. Owing to the different physical situations that aerosols provide, aerosols offer a simpler system in which to study coagulation than do colloids. In particular, particlesolvent interactions are absent. Furthermore, foreign coagulants need not be introduced and, therefore, particle-particle interactions can differ significantly from those found in colloids. Aerosols, moreover, are not constrained to the hydrodynamic regime and can offer a variety of Knudsen numbers where only a limited number of coagulation studies have been carried out. These regimes, beyond $\mathrm{K} n \sim 0$, offer potentially fertile fields of exploration.

In this paper, we have chosen to investigate the coagulation dynamics of a liquid-drop aerosol system just outside of the continuum limit, where $\mathrm{Kn}$ is significantly larger than zero. The average Knudsen numbers range from $\mathrm{Kn} \approx 0.26$ to 0.14 at the completion of the experiment. We have chosen a liquid-drop system where the 
aggregates retain their spherical symmetry during growth. Therefore we did not have to incorporate the fractal nature of the aggregate into our data analysis.

Our experimental method employs both static and dynamic light-scattering (DLS) techniques. The DLS allows us to measure the model radius $r_{M}$ of an assumed zeroth-order lognormal distribution (ZOLD). Absolute static-light-scattering measurements from the aerosol are achieved by calibrating against liquids with known Rayleigh ratios. The measured intensity combined with the assumed ZOLD and the DLS measured modal radius allows us to determine the aerosol number concentration $M_{0}$. Others have measured rate constants for similar aerosol systems [15-19]. Our method, however, makes an absolute rate constant measurement solely by optical means. Therefore our technique is not subject to the errors that were endemic to the previous methods.

As a check of our results, we have numerically solved, using a finite-difference technique, Smoluchowski's coagulation-rate equation for a system having initial conditions closely resembling our experimental ones. We have found good agreement between the numerical coagulation rate and those measured from five experimental runs. Our numerical results describing $\langle r(t)\rangle$ are also in agreement with the scaling behavior of $r_{M}(t)$ from an assumed ZOLD and experiment.

\section{BACKGROUND}

The fundamental description of irreversible coagulation dynamics is given by the Smoluchowski equation $[20,21]$

$$
\begin{aligned}
\frac{\partial n(u, t)}{\partial t}= & \frac{1}{2} \int_{0}^{u} K(u-v, v) n(u-v, t) n(v, t) d v \\
& -n(u, t) \int_{0}^{\infty} K(v, t) n(v, t) d v,
\end{aligned}
$$

where $n(u, t)$ is the number concentration of particles of volume $u$ at time $t$. The integrals describe the rate at which particles of volume $u$ appear (first integral) and the rate at which they are lost (second integral). The Smoluchowski equation is rigorous under the mean-field approximation, where fluctuations in $n(u, t)$ are neglected. Only binary collisions are being considered by Eq. (1), which for volume fractions less than $1 \%$ is a good approximation [22]. Our experimental volume fractions were quite small, less than $10^{-6}$.

The coagulation kernel $K(u, v)$ is a key parameter in Smoluchowski's equation. It describes the reaction rate between particles of volumes $u$ and $v$, which ultimately determines the characteristic particle distribution $n(u, t)$. Solutions to Smoluchowski's coagulation equation are dependent on the mathematical form of $K(u, v)$. Whereas exact solutions to Eq. (1) exist only for a few mathematically simple kernels, general scaling solutions for $n(u, t \rightarrow \infty)$ are well known [6,7]. For homogeneous kernels defined as

$$
K(a u, a v)=a^{\lambda} K(u, v),
$$

where $\lambda$ is the degree of homogeneity, the well-known scaling solutions to Eq. (1) are

$$
n(u, g)=n(u, t \rightarrow \infty)=A g(t)^{-2} \phi(u / g),
$$

where $\phi(x)$ depends on the dimensionless variable $x=u / g$ and has a shape independent of time. This dynamic scaling relation clearly states that all distributions, regardless of their initial shape, evolve into the same final shape. This shape is predicted by the form of $K(u, v)$. The time dependence of the scaling distribution, which we designate by $n(u, g)$ is carried solely by the parameter $g(t)$. The parameter $g$ may be set equal to any ratio of moments such that $g=M_{i+1} / M_{i}$ [see Eq. (4) below]. This form is consistent with the requirement that the total particulate volume of a closed system remains constant. Finally, the choice for $g$ fixes the normalization conditions for the scale function $\phi(x)$ and the constant $A$. Beyond this, the selection of $g$ is arbitrary. We choose $g$ to represent the mean cluster size, i.e., $g(t)=M_{1} / M_{0}(t)$, which consequently fixes $A=M_{1}$ and $\int_{0}^{+\infty} \phi(x) d x$ $=\int_{0}^{+\infty} x \phi(x) d x=1$.

Light-scattering experiments designed to probe coagulating media measure some combination of the moments of the particle-size distribution. These moments are defined by

$$
M_{i}(t)=\int_{0}^{\infty} u^{i} n(u, t) d u,
$$

where $M_{i}(t)$ is the $i$ th moment of the particle distribution at time $t$. Therefore light-scattering experiments involving particulate media require an understanding of the temporal behavior of the moments for a correct interpretation of the coagulation dynamics.

The time rate of change of the $i$ th moment is found by multiplying Eq. (1) by $u^{i}$ and integrating over all $u$ to yield

$$
\begin{aligned}
\frac{d M_{i}}{d t}=\frac{1}{2} \int_{0}^{\infty} \int_{0}^{\infty} & {\left[(u+v)^{i}-u^{i}-v^{i}\right] } \\
& \times K(u, v) n(u, t) n(v, t) d u d v .
\end{aligned}
$$

In the scaling regime, defined where Eq. (3) holds, the functional form of the temporal behavior of the moments is time invariant. We call such moments scaled moments and we will designate them as $M_{i}$. Using the homogeneity of the coagulation kernel, Eq. (2), and the scaling form of the particle distribution, Eq. (3), in Eq. (5) we obtain

$$
\frac{d M_{i}}{d t}=M_{1}^{2} g(t)^{i+\lambda-2} I_{i}(K, \phi),
$$

where

$$
\begin{aligned}
I_{i}(K, \phi)=\int_{0}^{\infty} \int_{0}^{\infty} & {\left[(x+y)^{i}-x^{i}-y^{i}\right] } \\
& \times K(x, y) \phi(x) \phi(y) d x d y
\end{aligned}
$$

and is also independent of time. Analogous to the scaled distribution function [see Eq. (3)], the dynamic behavior of the scaled moments is solely described by the same parameter $g$.

To find the explicit time dependence of $g$ we use $g(t)=M_{1} / M_{0}(t)$ and Eq. (6) with $i=0$ (recall that $M_{1}$ is a constant) to find 


$$
g(t)=g\left(t_{0}\right)\left[\left(t-t_{0}\right) / t_{c}+1\right]^{z},
$$

where $z=(1-\lambda)^{-1}$, and

$$
t_{c}=-2 z /\left[I_{0} M_{1}^{\lambda} M_{0}\left(t_{0}\right)^{1 / z}\right] .
$$

The dynamic exponent $z$ carries information concerning the kernel homogeneity, while $t_{c}$ is a measure of the characteristic coagulation rate. As we will see below, $t_{c}$ is directly related to the absolute coagulation rate constant. The initial time $t_{0}$ is any chosen time after the distribution has achieved a scaling form. Substituting the time dependence of $g(t)$ back into Eq. (6), we finally arrive at the expression describing the time evolution of the scaled moments [23],

$$
M_{i}(t)=M_{i}\left(t_{0}\right)\left[\left(t-t_{0}\right) / t_{c}+1\right]^{(i-1) z} .
$$

Ultimately, Eq. (10) will allow us to determine the dynamic exponent $z$, hence $\lambda$, and the characteristic coagulation time $t_{c}$ from our quasielastic light-scattering data. From $t_{c}$, we will further determine the real-time coagulation rate of our aerosol system (see below).

In the continuum limit, where the mean free path of the ambient gas molecules is short relative to the particle, i.e., $\mathrm{Kn} \rightarrow 0$, the dynamics of the coagulation is accurately described with the Brownian kernel which is given by [24]

$$
K(u, v)=K_{0} \beta(u, v),
$$

where $\beta(u, v)=\left(u^{1 / 3}+v^{1 / 3}\right)\left(u^{-1 / 3}+v^{-1 / 3}\right)$. The rate coefficient is $K_{0}=2 k_{B} T / 3 \eta$, where $k_{B}$ is Boltzmann's constant and $T$ and $\eta$ are the absolute temperature and shear viscosity of the ambient gas, respectively. The Brownian kernel has $\lambda=0$.

For Kn slightly larger than zero, however, the Brownian kernel must be modified, and this is done by introducing the Cunningham correction factor into the diffusional term of $\beta(u, v)$. The Cunningham correction is an empirical modification for the Stokes-Einstein diffusion coefficient. For coagulation outside of the continuum limit one has

$\beta(u, v)=\left(u^{1 / 3}+v^{1 / 3}\right)\left[C\left(r_{u}\right) u^{-1 / 3}+C\left(r_{v}\right) v^{-1 / 3}\right]$,

where $C\left(r_{u}\right)$ is the Cunningham correction factor given by [25]

$$
C\left(r_{u}\right)=1+1.257 \mathrm{Kn}+0.4 \exp (-1.1 / \mathrm{Kn}) .
$$

The Cunningham-corrected Brownian kernel is not homogeneous but for $\mathrm{Kn} \leq 1, C\left(r_{u}\right)$ varies slowly with $r_{u}$ $\left(u=4 / 3 \pi r_{u}^{3}\right)$ and we can make the additional approximation $C\left(r_{u}\right)=C\left(r_{v}\right)=C\left(r_{g}\right)$, where $r_{g}$ is the radius of the average particle volume $g$. Using this approximation, we write

$$
K(u, v)=K_{0} C\left(r_{g}\right) \beta(u, v)
$$

to retain homogeneity in the kernel representation. Notice that the rate coefficient is not only increased by the factor $C\left(r_{g}\right)$ but also becomes a slow function of time since $r_{g}$ is a function of time. We will justify, however, that this time dependence is indeed slow and it will not significantly affect our experimental results.

Using the approximation given by Eq. (14), we proceed and calculate the characteristic coagulation time $t_{c}$. Integration of $I_{i}(K, \phi)$ with $i=0$ and $K(x, y)$ given by Eq. (14) yields

$$
I_{0}=-2 K_{0} C\left(r_{g}\right)\left(1+m_{1 / 3} m_{-1 / 3}\right),
$$

where $m_{i}=\int x^{i} \phi(x) d x$. Now, using Eq. (9), we arrive at

$$
t_{c}=\left[K_{0} C\left(r_{g}\right)\left(1+m_{1 / 3} m_{-1 / 3}\right) M_{0}\left(t_{0}\right)\right]^{-1} \text {. }
$$

where $\lambda=0$. This choice for $\lambda$ is consistent with the homogeneity of the kernel derived above [see Eq. (14)].

To evaluate the $m_{1 / 3}$ and $m_{-1 / 3}$ moments we must assume a particle-size distribution. Lee has shown [26] analytically that a self-preserving (scaling) size distribution, obtained via Brownian coagulation, can be well approximated by a log-normal. In addition to Lee's work, Sorensen and Taylor have done a numerical study [27] concerning the light-scattering nature of the selfpreserving distribution during Brownian coagulation. These authors assumed a ZOLD represented the selfpreserving size distribution and showed that a particular ratio (not important for our purposes here) of the lightscattering moments approached a constant as the distribution approached its scaling form. Their use of a ZOLD, instead of the log-normal discussed by Lee, was somewhat arbitrary since identical results would have been obtained had they used the latter distribution. We also choose a ZOLD for our analysis below and show that the consequences of our choice do not influence the final results.

The ZOLD can be written as

$$
n(r)=\frac{N \exp \left(-1 / 2 \ln ^{2} \sigma_{r}\right)}{(2 \pi) r_{M} \ln \sigma_{r}} A^{\prime} \exp \left(\frac{-\ln ^{2}\left(r / r_{M}\right)}{2 \ln ^{2} \sigma_{r}}\right),
$$

where $A^{\prime}$ is determined by normalizing to $M_{1}$, the total mass of the system. The modal radius (directly proportional to the mean radius) is given by $r_{M}$ and $\sigma_{r}$ represents the geometric width of the distribution in $r$ space. Using the ZOLD, we find $m_{1 / 3} m_{1 / 3}=\ln ^{2} \sigma_{r}$, the same result obtained by Lee who used the log-normal distribution instead.

Under the assumption that the log-normal distribution is self-preserving, Lee analytically showed that continuum Brownian coagulation leads to $\sigma_{r}^{\infty}=1.32$, where $\sigma_{r}^{\infty}$ is the self-preserving value of the geometric width. Light-scattering experiments, however, weight the large- $r$ end of a distribution. The work of Sorensen and Taylor showed that under the assumption that the selfpreserving distribution is a ZOLD, light-scattering moments yield $\sigma_{r}^{\infty}=1.25$. The term $\left(1+m_{1 / 3} m_{-1 / 3}\right)=2.05$ and 2.08 for $\sigma_{r}^{\infty}=1.25$ and 1.32 , respectively-a variation of only $1.5 \%$. In our analysis we use $\sigma_{r}^{\infty}=1.25$ since our results were obtained using light-scattering techniques. We show below, however, that our data analysis is somewhat insensitive to our choice for $\sigma_{r}^{\infty}$.

Finally, since it is our desire to determine the coagula- 
tion rate constant from our measurement of $t_{c}$, we use Eq. (16) and our calculation of $m_{1 / 3} m_{-1 / 3}$ to obtain

$$
K_{0}^{\prime}=K_{0} C\left(r_{g}\right)=\left[2.05 t_{c} M_{0}\left(t_{0}\right)\right]^{-1} \text {. }
$$

\section{EXPERIMENT}

The aerosol was formed from dioctylphthalate (DOP). A 2\% DOP-in-ethanol solution was nebulized with a constant output atomizer (TSI model no. 3075). The liquid drops were passed into a partially heated glass tube where the evaporation of the aerosol and subsequent condensation of new drops, in the cooler region of the tube, took place. A small trace of anthracene was added to the DOP-in-ethanol solution, providing nucleation sites for the newly condensed droplets. This evaporation/condensation scheme allowed for the creation of a homogeneous and quasimonodisperse aerosol. The aerosol then passed through a charge neutralizer (TSI model no. 3012) to produce a bipolar charge distribution. The TSI generator was designed to produce a uniform distribution that was well approximated by a ZOLD having a geometric width of $\sigma_{r} \approx 1.2$.

The aerosol was fed into a scattering chamber where the coagulation took place. The chamber was an aluminum cylinder $25 \mathrm{~cm}$ in diameter and $75 \mathrm{~cm}$ tall with optically flat windows positioned at $0^{\circ}, 90^{\circ}$ and $180^{\circ}$. The inside of the aluminum scattering cylinder was painted a flat black to reduce unwanted wall reflections from possible scattered laser light. Furthermore, the aluminum provided an isothermal surface which we found necessary to suppress unwanted thermally induced convection currents in the aerosol. The chamber was filled with DOP aerosol for $4 \mathrm{~min}$ before it was sealed. This allowed ample time for filling since the aerosol flow rate was approximately $8 \mathrm{l} / \mathrm{min}$. The radii typically ranged from 0.23 to $0.4 \mu \mathrm{m}$, implying Knudsen numbers ranging anywhere from 0.26 to $0.14(l \approx 0.06 \mu \mathrm{m})$ for a given coagulation run.

The aerosol coagulated via Brownian motion. In addition to the decreasing number concentration via coagulation we also observed a slow loss of aerosol to the walls of the container. This behavior was observed visually in the incident laser beam within a few minutes after the chamber was sealed, as dark layers, indicating the absence of aerosol particles, developed near the walls. These voids extended a distance of 2 to $3 \mathrm{~mm}$ from the wall. Our early time scattering measurements, however, were not affected since our scattering volume $\left(\sim 1 \mathrm{~mm}^{3}\right)$ was positioned at the center of the aluminum cylinder, approximately $12 \mathrm{~cm}$ away from any depletion layer. Unfortunately, thermal convection eventually transported some of these voids into our scattering volume thus limiting our maximum experimental run times to $20 \mathrm{~min}$.

An argon-ion laser was used and operated at $\sim 10 \mathrm{~mW}$ with incident wavelengths of either $\lambda_{0}=5145$ or $4880 \AA$. The incident beam was vertically polarized and passed through the $0^{\circ}$ and $180^{\circ}$ optical windows, $40 \mathrm{~cm}$ from the bottom of the cylinder. The scattered radiation passed through the $90^{\circ}$ window and a Glan-Thomson polarizer, oriented to pass vertically polarized light, before being collimated with an 8-cm focal-length lens. The lens was chosen such that the image of the scattering volume, with unit magnification, was focused onto an adjustable slit with a width set at $1 \mathrm{~mm}$. The collected polarized light passed through the slit and onto the photocathode of the photomultiplier tube (PMT), $50 \mathrm{~cm}$ behind the slit. This optical arrangement allowed for the proper degree of spatial coherence on the cathode necessary for a good signal-to-noise ratio. The photopulses from the PMT were amplified and discriminated before being fed into a commercial correlator. The correlator was used to calculate both total scattered intensity and the homodyne intensity autocorrelation function.

Two types of measurements were made during a typical light-scattering experiment. First, the mean droplet size was determined from the diffusion coefficient measured with the intensity autocorrelation function [28]. This correlation function in the homodyne detection mode is given by [29]

$$
\left\langle I_{v}(0) I_{v}(t)\right\rangle=B+A \exp \left(-\mu_{1} t+1 / 2 \mu_{2} t^{2}\right),
$$

where $I_{v}$ denotes vertically polarized scattered light. In Eq. (19), $B$ is a background determined either with the photocount statistics or the long time $(t \rightarrow \infty)$ behavior of the autocorrelation function. $A$ is the amplitude of the signal. A fit of our data to Eq. (19) yielded the first and second cumulants, $\mu_{1}$ and $\mu_{2}$. The cumulants are related to the mean particle size and geometric width of the size distribution, respectively. In this work, however, no quantitative effort was made to determine the width of our particle size distributions. Given the evolution of our system, accumulation times for $\left\langle I_{v}(0) I_{v}(t)\right\rangle$ were necessarily short, hence sufficient statistics for an accurate determination of $\mu_{2}$ could not be obtained. Therefore we included $\mu_{2}$ only as a fitting parameter to increase the accuracy of $\mu_{1}$.

The first cumulant contains the desired mean particlesize information through

$$
\mu_{1}=2 D q^{2}
$$

where the scattering wave vector in the quasielastic limit is $q=4 \pi \lambda^{-1} \sin (\theta / 2)$. The experimental scattering angle was fixed for all coagulation runs at $\theta=90^{\circ}$. Also in Eq. (20) is the particle diffusion coefficient

$$
D=\frac{C(r) k_{B} T}{6 \pi \eta r},
$$

where $C(r)$ is the previously defined Cunningham correction factor modifying the Stokes-Einstein diffusion coefficient $k_{B} T / 6 \pi \eta r$ [see Eq. (13)]. Of course, our system cannot be monodisperse and the diffusion coefficient extracted from the fit to the correlation function is really an average over the entire size distribution. In fact, $\mu_{1}$ is a ratio of moments of the particle-size distribution. The actual procedure used to extract the mean radius from the experimental $\mu_{1}$ is described below.

The second quantity determined during a given run was the vertically polarized scattering intensity $I_{v}$. Since details such as the size of the scattering volume, the PMT 
efficiency, and the solid collection angle $d \Omega$ are difficult to measure, a quantitative measurement of the absolute scattered intensity was obtained by a simple calibration technique using liquids with known Rayleigh ratios. Four liquids were used, benzene $\left(\mathrm{C}_{6} \mathrm{H}_{6}\right)$, toluene $\left(\mathrm{C}_{7} \mathrm{H}_{8}\right)$, carbon tetrachloride $\left(\mathrm{CCl}_{4}\right)$, and carbon disulfide $\left(\mathrm{CS}_{2}\right)$. The Rayleigh ratios are functions of both the incident wavelength and the incident and scattered polarizations. We have used the Rayleigh ratios measured by Moreels, DeCeuninck, and Finsy [30] and Coumou et al. [31-33] for the case of vertically polarized incident and scattered radiation at $\theta=90^{\circ}$.

Each calibration liquid was of spectroscopic grade. The liquids were loaded into glass cylinders $7 \mathrm{~cm}$ in diameter and $12 \mathrm{~cm}$ in length and sealed under a nitrogen atmosphere glove box to prevent any contamination from water and foreign particulates. The liquid cells were subsequently centered in the aerosol scattering chamber to obtain normal incidence of the laser beam and the exact $90^{\circ}$ scattering angle. The scattered intensities were then measured for each of the calibration liquids. This scattered intensity is given by [34]

$$
I_{v}=\Phi\left(I_{0 v}, V_{s}, d \Omega, \epsilon\right) R_{v v},
$$

where $R_{v v}$ is Rayleigh ratio (see Table I). The constant $\Phi\left(I_{0 v}, V_{s}, d \Omega, \epsilon\right)$ is the calibration constant dependent on the vertically polarized incident intensity $I_{0 v}$, the scattering volume $V_{s}$, the collection angle $d \Omega$, and the PMT efficiency $\epsilon$. Refraction of the slightly focused incident beam in the liquids changes both the scattering volume and the incident intensity relative to the aerosol chamber, but in such a manner that their product, which is in $\Phi$, remains invariant. $\Phi$ determined in this manner, therefore, may be used for calibrating the absolute scattered intensity.

Equation (22) shows that $\Phi\left(I_{0 v}, V_{s}, d \Omega, \epsilon\right)$ is simply the slope of an $I_{v}$ vs $R_{v v}$ plot. Thus a useful average for $\Phi$ is obtained using the $I_{v}$ measurements of the calibration liquids. An example of a typical calibration run is given by Fig. 1. The best-fit lines, forced to pass through the origin, had associated uncertainties for $\Phi$ of $\pm 5 \%$ for our five experimental runs. Furthermore, we have tested our calibrations against polystyrene suspensions $(0.232$ and $0.091 \mu \mathrm{m}$ ) of known particle concentrations. We found excellent agreement between these known concentrations and the calculated particle concentrations using $\Phi$ (see Fig. 2). The actual determination of the number concentration of the aerosol from $\Phi$ is discussed in detail below.

A typical run began with the intensity calibration fol-

TABLE I. The Rayleigh ratios used for calibrating the scattering geometry and the instrument sensitivities. See Refs. [30-34].

\begin{tabular}{lcc}
\hline Liquid & $R_{v v}\left(90^{\circ}\right), \lambda_{0}=4880 \AA$ & $R_{v v}\left(90^{\circ}\right), \lambda_{0}=5145 \AA$ \\
\hline $\mathrm{C}_{6} \mathrm{H}_{6}$ & $29.1 \times 10^{-6} \mathrm{~cm}^{-1}$ & $23.0 \times 10^{-6} \mathrm{~cm}^{-1}$ \\
$\mathrm{C}_{7} \mathrm{H}_{8}$ & $31.0 \times 10^{-6} \mathrm{~cm}^{-1}$ & $24.2 \times 10^{-6} \mathrm{~cm}^{-1}$ \\
$\mathrm{CCl}_{4}$ & $17.1 \times 10^{-6} \mathrm{~cm}^{-1}$ & $13.5 \times 10^{-6} \mathrm{~cm}^{-1}$ \\
$\mathrm{CS}_{2}$ & $111.0 \times 10^{-6} \mathrm{~cm}^{-1}$ & $82.2 \times 10^{-6} \mathrm{~cm}^{-1}$ \\
\hline \hline
\end{tabular}

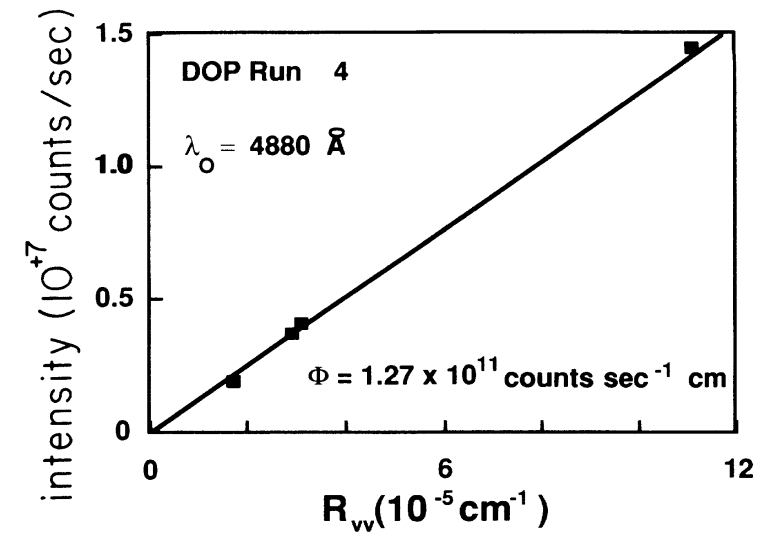

FIG. 1. A typical calibration run for calibrating the scattering geometry and instrument sensitivities against liquids with known Rayleigh ratios (see Table I).

lowed by the filling of the aerosol chamber (careful not to perturb the collection optics) with the DOP aerosol. Intensity and intensity autocorrelation measurements began immediately after the fill. Aerosol coagulation during the duration of the fill could obviously not be avoided. Fortunately, scaling distributions are insensitive to linear transformations in time, hence $t=0$ can be defined at any time $t_{0}$ once scaling has been achieved. To obtain values of the first cumulant with accuracy sufficient for our purpose, accumulation times of $40 \mathrm{sec}$ were desirable for measuring $\left\langle I_{v}(0) I_{v}(t)\right\rangle$. These run times, moreover, were short enough to avoid averaging effects which occurred due to the forever changing nature of our coagulating aerosol. The scattered intensity $I_{v}$ was measured essentially instantaneously. The runs were limited to 10-20 min durations owing to the convection of the wall depletion layers into the scattering volume as already de-

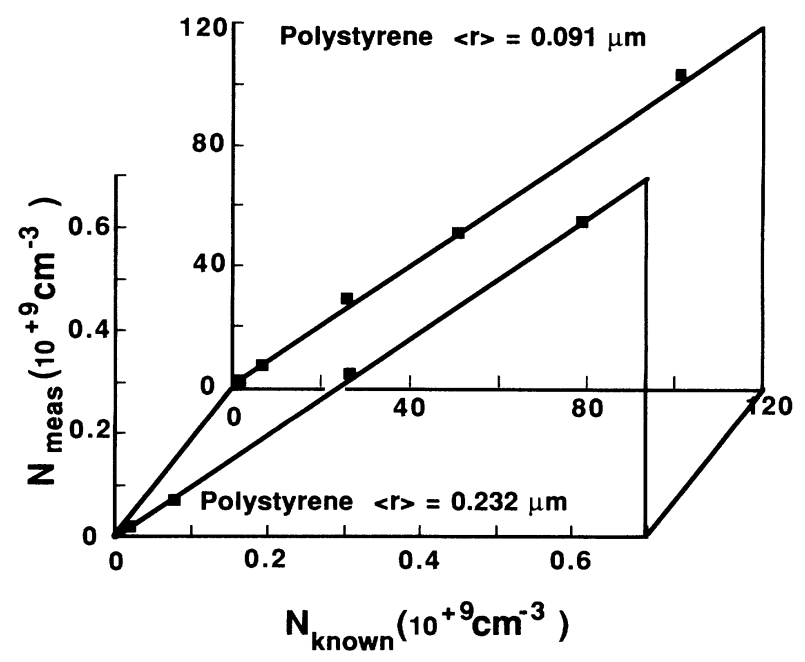

FIG. 2. Testing the derived calibration constant: Plotted are the measured number concentrations (calculated using a derived calibration constant) vs the known number concentrations from prepared suspensions of polystyrene latex spheres. 
scribed above. Furthermore, this limit was easily determined with the eye and found to correlate well with fluctuations in the scattered intensities. After the onset of these fluctuations, quantities dependent on $I_{v}$ were significantly changed. We point out, however, that although absolute intensity measurements were affected by propagating voids through the scattering volume, the intensity autocorrelation function was not. In fact, the shape of $\left\langle I_{v}(0) I_{v}(t)\right\rangle$ was not visually influenced until a significant number of particles began to gravitationally settle out from the scattering volume. After the completion of the aerosol coagulation, a second calibration run was performed which always agreed with the first to within $3 \%$.

\section{DATA ANALYSIS AND RESULTS}

The general outline of the data analysis was the following: (1) to determine the modal radius $r_{M}$ of an assumed ZOLD size distribution as a function of time from the measured homodyne autocorrelation function, and (2) using $r_{M}$, the assumed ZOLD, and the measured scattered intensity $I_{v}$, calculate the number concentration $M_{0}$ as a function of the coagulation time. Then, the characteristic coagulation time $t_{c}$, hence $K_{0}^{\prime}$, is found from the dynamic behavior of both $r_{M}(t)$ and $M_{0}(t)$.

\section{A. The model radius $r_{M}$}

The well-known mathematical expression relating the first cumulant to the size distribution is

$$
\mu_{1}^{\text {theor }}=\frac{\int_{0}^{\infty} \mu_{1}(r) n\left(r_{M}, \sigma_{r} ; r\right) i_{v}(r, \theta) d r}{\int_{0}^{\infty} n\left(r_{M}, \sigma_{r} ; r\right) i_{v}(r, \theta) d r},
$$

where $\mu_{1}(r)$ is the first cumulant for a particle of radius $r$ as given by Eq. (20). Also in the integrand, $i_{v}(r, \theta)$ represents the scattered-light intensity of a particle of radius $r$ at the given experimental scattering angle of $\theta=90^{\circ}$. For our particle sizes, $i_{v}(r, \theta)$ must be calculated with the Mie algorithm [35]. Finally, the assumed ZOLD $n\left(r_{M}, \sigma_{r} ; r\right)$ is given by Eq. (17). To determine the dynamic behavior of $r_{M}(t)$, we first fit the measured $\left\langle I_{v}(0) I_{v}(t)\right\rangle$ to obtain $\mu_{1}^{\text {expt }}$ and then minimize the difference $\delta=\mu_{1}^{\text {theor }}-\mu_{1}^{\text {expt }}$ by numerically integrating Eq. (23) while sequentially varying $r_{M}$ until the minimum is found. Following this procedure at different times during a coagulation experiment yields $r_{M}(t)$.

As discussed above, the value we choose to represent the self-preserving width is the one obtained via the light scattering studies, i.e., $\sigma_{r}^{\infty}=1.25$. We have, however, studied the effect of a variation of $\sigma_{r}^{\infty}$ in a range between our estimated starting value of 1.20 and the analytical value derived by Lee, $\sigma_{r}^{\infty}=1.32$. Choosing a reference value, $\sigma_{\text {ref }}=1.25$, and an arbitrary value for $r_{M}$, we numerically solve Eq. (23) for $\mu_{1}^{\text {theor }}\left(\sigma_{\text {ref }}\right)$. Then, to estimate the effect $\sigma_{r}^{\infty}$ will have on our determination of $r_{M}$, we increment it to other values in the range $1.20-1.32$ and minimize the difference $\delta=\mu_{1}^{\text {theor }}\left(\sigma_{\text {ref }}\right)-\mu_{1}^{\text {theor }}\left(\sigma_{r}^{\infty}\right)$ again by varying $r_{M}$. The results of the calculations are plotted in Fig. 3. The variation over the entire $\sigma_{r}^{\infty}$ range is only

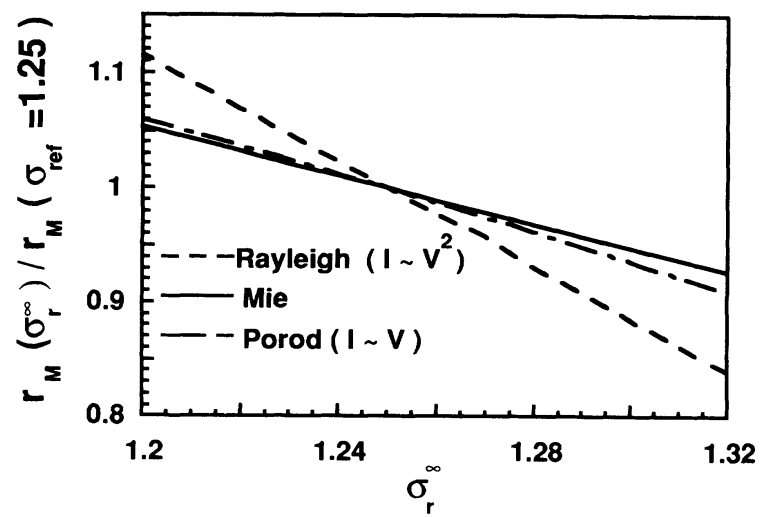

FIG. 3. Variation of the modal radius $r_{M}$ with the geometric, self-preserving width $\sigma_{r}^{\infty}$.

$13 \%$, which is acceptable for our analysis below.

It is interesting to note that in the Mie-scattering regime where $\theta=90^{\circ}, r_{M}$ shows the weakest dependence on $\sigma_{r}^{\infty}$. This weak dependence probably results from the angular dependence of the scattered radiation. Conversely, in the Rayleigh regime where the scattered intensity is isotropic, and $I_{v} \propto r^{6}\left(v^{2}\right)$, the strongest dependence on $\sigma_{r}^{\infty}$ is observed. Because all of our aerosol systems are scattering in the Mie regime, Fig. 3 confirms that our analysis for $r_{M}$ will not be overly sensitive to our choice of $\sigma_{r}^{\infty}$.

For $\mathrm{Kn} \rightarrow 0$, Eq. (23) shows that $\mu_{1}$ is related to the moments of the particle-size distribution. Using Eqs. (10) and (23) one can show

$$
r_{M}(t)=r_{M}\left(t_{0}\right)\left[\left(t-t_{0}\right) / t_{c}+1\right]^{z / 3}
$$

since, by Eqs. (20) and (21), $\mu_{1}^{-1} \sim r \sim u^{1 / 3}$. Equation (24) predicts that a $\log$-log plot of $r_{M}$ vs $t$ will approach linearity as $t \gg t_{c}$. Alternatively, a $\log$ plot of $r_{M}$ vs $t+t_{c}$ should be linear for the proper value of $t_{c}$. The dynamics of $r_{M}(t)$, found from minimizing $\delta=\mu_{1}^{\text {theor }}-\mu_{1}^{\text {expt }}$, are given in Figs. 4 and 5 where $t_{0}=0$ represents the start of our data collection. By setting $t_{0}=0$ we have some-

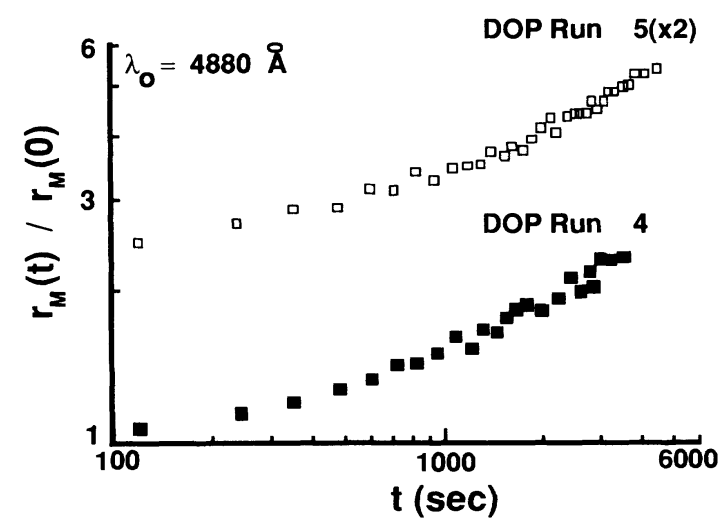

FIG. 4. Log-log plot of time evolution of the experimentally derived $r_{M}$ (from an assumed ZOLD with $\sigma_{r}^{\infty}=1.25$ ) for two representative coagulation runs. 


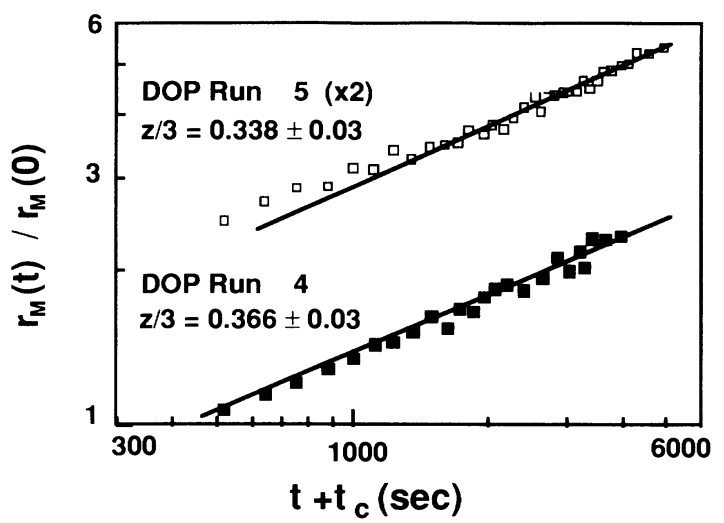

FIG. 5. Data from Fig. 4 only the independent variable is $t+t_{c}$. The characteristic coagulation time $t_{c}$ was varied until a best fit to linearity was achieved.

what boldy assumed that our aerosol size distribution has obtained a self-preserving form. This assumption is not totally unfounded since at least $4 \mathrm{~min}$ of aerosol coagulation takes place while the chamber is being filled and the initial width $\sigma_{r} \approx 1.2$ is itself not far from the actual scaling value.

Figure 4 shows log-log plots of $r_{M}$ vs $t$ for coagulation runs 4 and 5 . Both clearly show an approach to linearity as $t$ becomes large. Alternatively, Fig. 5 shows the corresponding log-log plots of $r_{M}$ vs $t+t_{c}$. For the data in Fig. $5, t_{c}$ was varied until a best fit to linearity was achieved. The linearity was our criterion for determining $t_{c}$ from $r_{M}(t)$. Figure 5 gives the representative coagulation runs that provide the upper and lower limits for the experimental scaling exponent $z / 3$. For the five experimental runs, we found $\langle z / 3\rangle=0.36 \pm 0.2$.

For Brownian coagulation, $z / 3=0.333$ since $z=1$ $(\lambda=0)$. Unfortunately, the Cunningham corrected Brownian kernel is not homogeneous, but the dynamic behavior of $r_{M}(t)$ shown in Figs. 4 and 5 suggests an effective homogeneity of $\lambda_{\text {eff }}=0.07 \pm 0.06$. This value is not unreasonable since our experimental conditions are just outside of the Brownian coagulation limit and since $\lambda$ is expected to increase to $\frac{1}{6}$ in the $\mathrm{Kn} \rightarrow \infty$ limit. We point out, however, that one must be cautious in interpreting $\lambda_{\text {eff }}$ as a true kernel homogeneity since the Knudsen number is continuously changing during any given run. In fact, Knudsen numbers varied by as much as $40 \%$. As a consequence of the changing Knudsen number, we predict a time-dependent $t_{c}$ [see Eq. (16)]. Despite a changing $t_{c}$, our fitting procedure has forced linearity upon the data in Fig. 5 when the data should have appeared curved. The suppressed curvature consequently leads to an increased value for $z$ (hence $\lambda$ ). The increase, however, is within our experimental error for determining $z$ and we conclude that $\lambda \approx 0$, and this result supports our earlier approximation to the Cunningham corrected Brownian kernel [see Eq. (14)].

As Figs. 4 and 5 illustrate, the behavior of $r_{M}(t)$ is well represented by Eq. (24), and therefore our assumption of a scaling distribution at the start of data collection is justified. The scaling exponents $z / 3$ are easily determined by linearizing $r_{M}(t)$ with respect to the characteristic coagulation time $t_{c}$. The best-fit values for the $t_{c}$ 's in Fig. 5 are $400 \pm 50 \mathrm{sec}$. However, because of the changing nature of $\mathrm{Kn}$, hence $t_{c}$, our calculated values for $t_{c}$ must be further questioned.

Unfortunately, we could not predict $t_{c}$ from the dynamic behavior of $r_{M}(t)$ from aerosol runs $1-3$ because of relatively short run-time durations. Consequently, the shortened time intervals would not allow for a large enough dynamical range in $r_{M}(t)$ and our plots of $r_{M}(t)$ vs $t+t_{c}$ were insensitive to any meaningful variation in $t_{c}$. We describe, below, another approach for determining more reliable $t_{c}$ 's for our aerosol systems.

As a test of the analysis given above, the Smoluchowski coagulation equation was numerically solved under initial conditions closely resembling our experimental ones. That is, an initial ZOLD was assumed, with $\sigma=1.2, \quad r_{M}(0)=0.26 \mu \mathrm{m} \quad$ and $\quad M_{0}(t)=1.0 \times 10^{7}$ particles/cc. The initial ZOLD evolved in real time, according to the Smoluchowski equation, to a time equivalent to our experimental run times. The dynamic behavior of $\left\langle{ }_{i}(t)\right\rangle$, resulting from the numerical solution, is plotted in Fig. 6. Because the ZOLD is not a true solution to Smoluchowski's equation, we derive an average hydrodynamic radius from the first cumulant to test our experimental results instead of using $r_{M}$. The numerical results show that the temporal behavior of $\langle r(t)\rangle$ is accurately described with Eq. (24) and the dynamic scaling exponent $z / 3=0.38$ is in good agreement with our experimental results.

\section{B. The number density $M_{0}(t)$}

Our second method of analysis is to use the measured scattered intensity to determine the aerosol number den-

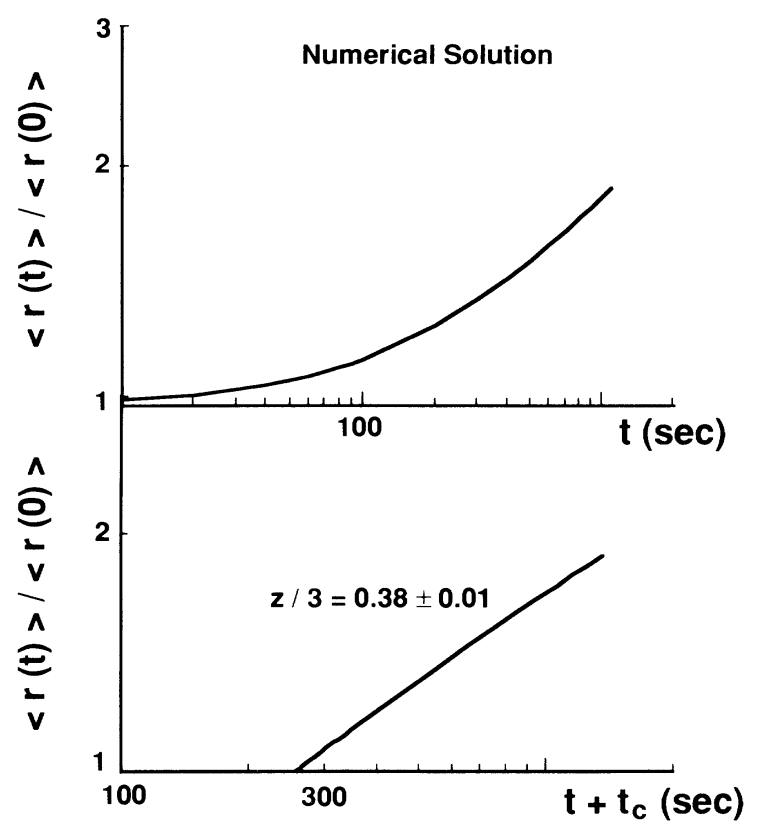

FIG. 6. Log-log plot of the dynamic behavior of the average hydrodynamic radius according to Smoluchowski's equation. 


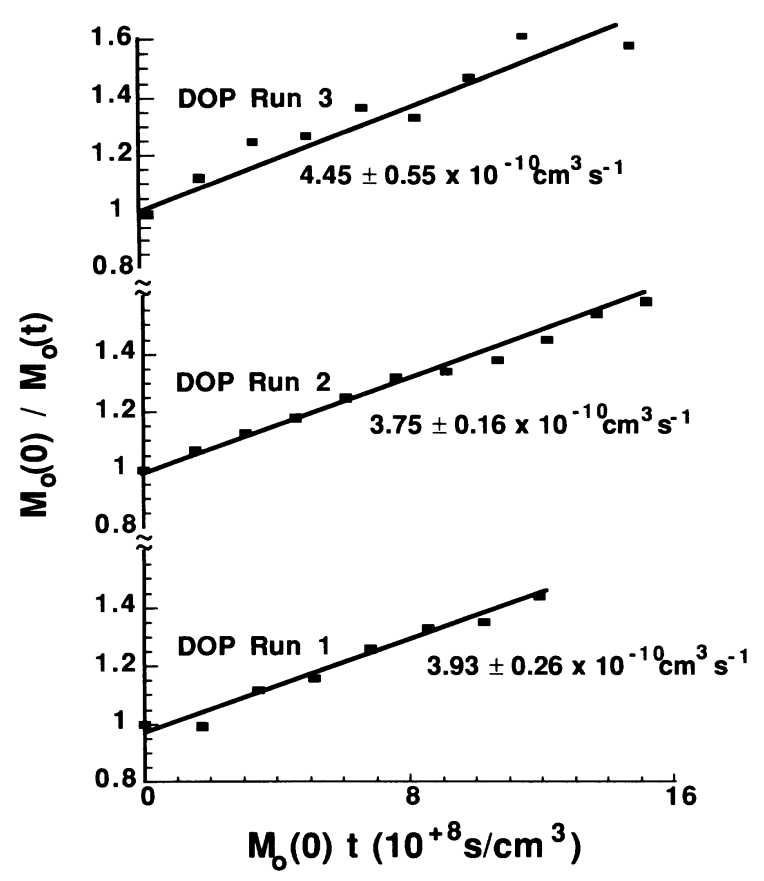

FIG. 7. Time evolution of the number concentration $M_{0}(t)$ for coagulation runs 1,2 , and 3. These runs used $\lambda_{0}=5145 \AA$. The given slopes are equal to $2.05 K_{0}^{\prime}$.

sity $M_{0}(t)$. If the distribution has a scaling form, the temporal behavior of $M_{0}(t)$ [i.e., $\mathcal{M}_{0}(t)$ ] will yield $t_{c}$ hence $K_{0}^{\prime}$.

The total scattered intensity at time $t$ is given by

$$
I_{v}(t)=M_{0}(t) \Phi \int_{0}^{\infty} n\left[r_{M}(t), \sigma ; r\right] i_{v}(r, \theta) d r
$$

To determine $M_{0}(t)$ from $I_{v}(t)$ we once again assume a ZOLD size distribution with $\sigma_{\infty}=1.25$. For $r_{M}(t)$, we use the values found from minimizing $\delta=\mu_{1}^{\text {theor }}-\mu_{1}^{\text {expt }}$ and $i_{v}(r, \theta)$ is calculated using the Mie algorithm. $\Phi$ is the calibration constant determined from the Rayleigh ratios before and after each experimental run. Numerical inversion of Eq. (25) gives us $\boldsymbol{M}_{0}(t)$.

If our aerosol distribution is self-preserving, then from Eqs. (10) and (17) we predict for the scaled moments

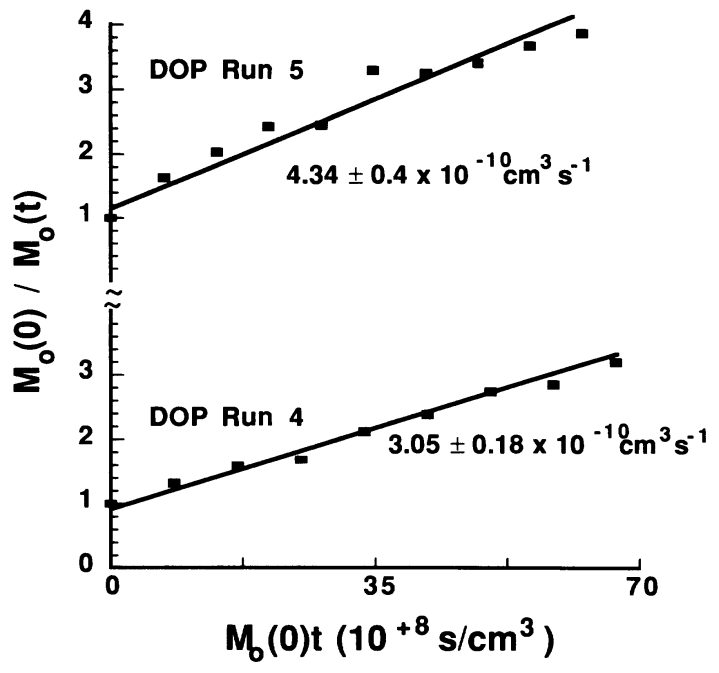

FIG. 8. Time evolution of the number concentration $M_{0}(t)$ for coagulation runs 4 and 5. These runs used $\lambda_{0}=4880 \AA$. The slopes equal $2.05 K_{0}^{\prime}$.

$$
\frac{M_{0}(0)}{M_{0}(t)}=2.05 K_{0}^{\prime} M_{0}(0) t+1,
$$

where $\lambda=0$. Equation (26) suggests a plot of $M_{0}(0) / M_{0}(t)$ vs $M_{0}(0) t$ is linear with a slope equal to $2.05 K_{0}^{\prime}$ and an intercept of 1 . The results of our five coagulation runs are plotted in Figs. 7 and 8 . The observed linearity for all five runs suggests a self-preserving shape for the aerosol distributions. From a least-squares fitting procedure, we find for our five runs an average coagulation rate constant of $\left\langle K_{0}^{\prime}\right\rangle=1.95 \pm 0.17 \times 10^{10}$ $\mathrm{cm}^{3} \mathrm{~s}^{-1}$. The independent results for individual coagulation runs are summarized in Table II and are discussed in more detail below.

Using the measured values of $K_{0}^{\prime}$ from coagulation runs 4 and 5 along with Eq. (18), we calculate characteristic coagulation times of $t_{c}=405$ and $410 \mathrm{sec}$, respectively. These characteristic times are in good agreement with the values $400 \pm 50 \mathrm{sec}$ found from linearizing the temporal behavior of $r_{M}(t)$ by varying $t_{c}$ in Fig. 5 .

TABLE II. Summary of the experimental results.

\begin{tabular}{cccccccc}
\hline Run & $\left\langle r_{M}\right\rangle(\mu \mathrm{m})$ & $\langle\mathrm{Kn}\rangle$ & $\left\langle C\left(r_{M}\right)\right\rangle$ & $M_{0}(0)\left(\mathrm{cm}^{3}\right)$ & $\left\langle t_{c}\right\rangle(\mathrm{sec})$ & $\begin{array}{c}K_{0}^{\prime} \\
\left(\mathrm{cm}^{3} \mathrm{sec}^{-1}\right)\end{array}$ & $\begin{array}{c}K_{0}^{\prime}(\mathrm{theor}) \\
\left(\mathrm{cm}^{3} \mathrm{sec}^{-1}\right)\end{array}$ \\
\hline 1 & 0.29 & 0.23 & $1.29 \pm 0.045$ & $2.9 \times 10^{6}$ & 900 & $\begin{array}{c}(1.92 \pm 0.13) \\
\times 10^{-10}\end{array}$ \\
2 & 0.31 & 0.21 & $1.27 \pm 0.06$ & $2.5 \times 10^{6}$ & 1050 & $\begin{array}{c}(1.83 \pm 0.08) \\
\times 10^{-10}\end{array}$ \\
3 & 0.28 & 0.24 & $1.30 \pm 0.045$ & $2.7 \times 10^{6}$ & 830 & $\begin{array}{c}(2.17 \pm 0.27) \\
\times 10^{-10}\end{array}$ & $(1.92 \pm 0.09) \times 10^{-10}$ \\
4 & 0.42 & 0.16 & $1.20 \pm 0.13$ & $7.0 \times 10^{6}$ & 405 & $\begin{array}{c}(1.73 \pm 0.09) \\
\times 10^{-10}\end{array}$ & $(1.81 \pm 0.20) \times 10^{-10}$ \\
5 & 0.375 & 0.18 & $1.22 \pm 0.13$ & $5.6 \times 10^{6}$ & 410 & $\begin{array}{c}(2.12 \pm 0.20) \\
\times 10^{-10}\end{array}$ & $(1.84 \pm 0.20) \times 10^{-10}$ \\
Smoluchowski & 0.39 & 0.17 & $1.21 \pm 0.13$ & $1.0 \times 10^{7}$ & 255 & $\begin{array}{c}1.85 \times 10^{-10} \\
(1.83 \pm 0.20) \times 10^{-10}\end{array}$ \\
\hline \hline
\end{tabular}


We have used average Knudson numbers to estimate the coagulation rate constants predicted by theory for our experimental runs. From the initial and final values derived for $r_{M}$, we can calculate the averages $\left\langle r_{M}\right\rangle$, $\langle\mathbf{K n}\rangle$, and $\left\langle C\left(r_{M}\right)\right\rangle$. The average values of these quantities for each coagulation run are given in Table II. Now, by defining $K_{0}^{\prime}(t h)=\left\langle C\left(r_{M}\right)\right\rangle K_{0}=\left\langle C\left(r_{M}\right)\right\rangle 1.51$ $\times 10^{-10}$, we calculate the expected absolute coagulation rates for our five experimental runs. The expected values along with the experimental ones $\left(K_{0}^{\prime}\right)$ are found for comparison in Table II. The uncertainties given for $\left\langle C\left(r_{M}\right)\right\rangle$ and $K_{0}^{\prime}(t h)$ resulted from the range in $\mathrm{Kn}$ and are small (4-11\%), usually smaller than the experimental uncertainties in $K_{0}^{\prime}$. The expected and experimental values agree within the error.

Figure 9 shows the numerical real-time evolution of $M_{0}$ as derived from our numerical solution to Eq. (1). Again, an initial ZOLD was assumed, with $\sigma=1.2$, $r_{M}(0)=0.26 \mu \mathrm{m}$, and $M_{0}(t)=1.0 \times 10^{7}$ particles $/ \mathrm{cc}$ and allowed to evolve according to the Smoluchowski equation to a time equivalent to our experimental run times. The slope of the line in Fig 9 is equal to $a K_{0}^{\prime}$ $=3.925 \times 10^{-10} \mathrm{~cm}^{3} \mathrm{sec}^{-1}$ where $a=\left(1+m_{1 / 3} m_{-1 / 3}\right)$. We had previously assumed a ZOLD to evaluate these moments. Here, however, we do not know the mathematical form of the size distribution so we must evaluate $a$ numerically. We find $a=2.10$, therefore, $K_{0}^{\prime}=1.87 \times 10^{-10} \mathrm{~cm}^{3} \mathrm{sec}^{-1}$. This value is in good agreement with the values of $K_{0}^{\prime}(t h)$ found in Table II.

We now investigate the changes that would occur in the experimental coagulation rate $K_{0}^{\prime}$ if $\sigma_{\infty} \neq 1.25$. Again we choose $\sigma_{\text {ref }}=1.25$ and a corresponding value for $K_{0}^{\prime}\left(\sigma_{\text {ref }}\right)=1.51 \times 10^{-10} \mathrm{~cm}^{3} \mathrm{sec}^{-1}$ (the expected value in the continuum limit). $K_{0}^{\prime}\left(\sigma_{\text {ref }}\right)$ allows us to use Eqs. (24) and (26) to find $r_{M}\left(t ; \sigma_{\text {ref }}\right)$ and $M_{0}\left(t ; \sigma_{\text {ref }}\right)$ over a representative coagulation time interval of $\approx 1100 \mathrm{sec}$. Then using $r_{M}$ and $M_{0}$ at a particular time within the 1000 -sec duration, we numerically integrate Eq. (25) for $I_{v}\left(t ; \sigma_{\text {ref }}\right)$. Now to determine the dependence of $K_{0}^{\prime}$ on $\sigma_{r}^{\infty}$ we increment $\sigma_{r}$ within the range 1.20 to 1.32 and for

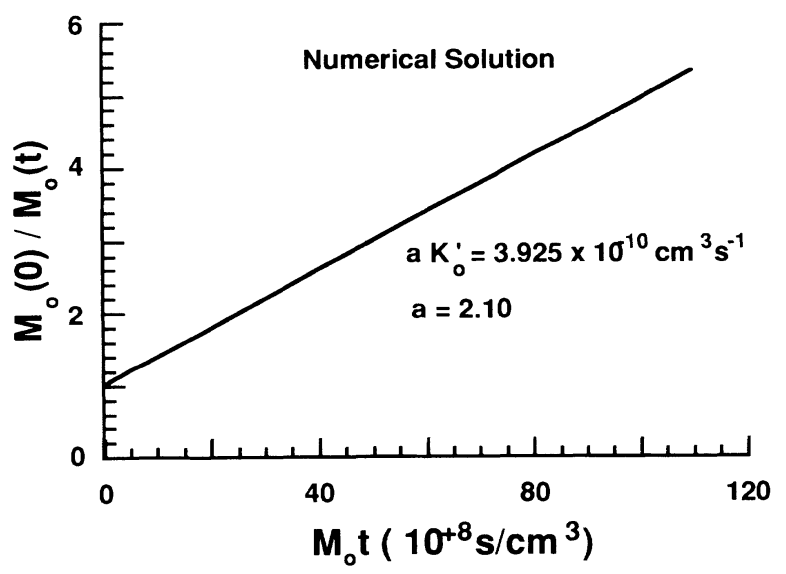

FIG. 9. Time evolution of the number concentration $M_{0}(t)$ according to numerical integration of Smoluchowski's equation. The value of the constant $a$ was found numerically also.

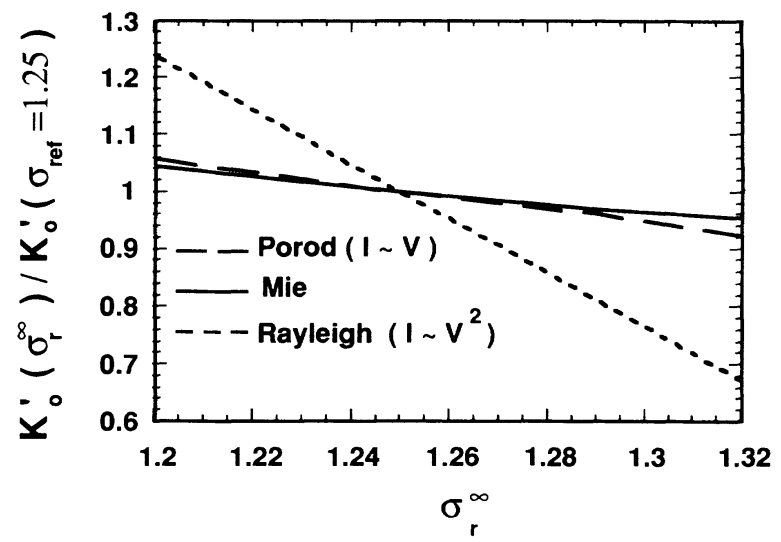

FIG. 10. Variation of the coagulation rate constant $K_{0}^{\prime}$ with the geometric, self-preserving width $\sigma_{r}^{\infty}$.

each increment solve for

$$
M_{0}\left(t ; \sigma_{r}^{\infty}\right)=\frac{I_{v}\left(t ; \sigma_{\mathrm{ref}}\right)}{\int_{0}^{\infty} n\left[r_{M}(t), \sigma ; r\right] i_{v}(r, \theta) d r},
$$

where $r_{M}\left(t ; \sigma_{r}^{\infty}\right)$ is determined using Fig. 3. Finally, we use the $M_{0}\left(t ; \sigma_{r}^{\infty}\right)$ for a given $\sigma_{r}^{\infty}$ and Eq. (26) to determine $K_{0}^{\prime}\left(\sigma_{r}^{\infty}\right)$ in the same manner as we did for the real data. Our results are plotted in Fig. 10. As $\sigma$ varies through the range $1.20-1.32, K_{0}^{\prime}$ varies only $9 \%$. Since this variation is on the order of our experimental and theoretical uncertainties, we conclude no significant change would occur by choosing a value other than $\sigma_{r}^{\infty}=1.25$.

\section{CONCLUSION}

We have studied the coagulation dynamics of a liquiddrop aerosol. Our aerosol systems were significantly into the transition regime between the Brownian and kinetic limits with mean Knudsen numbers of $\approx 0.2$. Our experimental method used a combination of static and dynamic light-scattering to determine the temporal behavior of the modal radius $r_{M}(t)$ and the number concentration $M_{0}(t)$ of an assumed ZOLD. We have found that the scaling theory predicts the dynamic behavior of the moments reasonably well.

From the scaling theory, we calculated the kernel homogeneity $\lambda$ and, most importantly, the absolute coagulation rate $K_{0}^{\prime}$ of our coagulating systems. Both $K_{0}^{\prime}$ and $\lambda$ agreed well with theory and a real-time numerical solution to Smoluchowski's coagulation equation.

We have found the assumption of a ZOLD was not critical to representing the true particle-size distribution since our analysis was in agreement with the numerical solution to Smoluchowski's equation. The assumed ZOLD was relatively insensitive to the self-preserving width $\sigma_{r}^{\infty}$.

Our experimental methods were the first to measure $K_{0}^{\prime}$ 
in a coagulating aerosol using only optical perturbations hence minimizing external influences which could have affected the results. It was the time-invariant nature of our aerosol particle distribution that allowed for the determination of $K_{0}^{\prime}$.

\section{ACKNOWLEDGMENTS}

We thank Kent Heady for help with some of the experiments. This work was supported by NSF Grant No. CBT8709622.
*Present address: LANSCE, MSH805, Los Alamos National Laboratory, Los Alamos, NM 87545.

[1] T. A. Witten and L. M. Sander, Phys. Rev. Lett. 47, 1400 (1981).

[2] P. Meakin, Phys. Rev. Lett. 51, 1119 (1983).

[3] S. K. Friedlander, J. Meteor. 18, 753 (1961).

[4] D. L. Swift and S. K. Friedlander, J. Colloid Interface Sci. 19, 621 (1964).

[5] C. S. Wang and S. K. Friedlander, J. Colloid Interface Sci. 24, 170 (1967).

[6] A. A. Lushnikov, J. Colloid Interface Sci. 45, 549 (1973).

[7] P. G. J. van Dongen and M. H. Ernst, Phys. Rev. Lett. 54, 1396 (1985).

[8] D. W. Schaefer, J. E. Martin, P. Wiltzius, and D. S. Cannell, Phys. Rev. Lett. 52, 2371 (1984).

[9] J. E. Martin and D. W. Schaefer, Phys. Rev. Lett. 53, 2457 (1984).

[10] D. A. Weitz, J. S. Huang, M. Y. Lin, and J. Sung, Phys. Rev. Lett. 53, 1657 (1984).

[11] B. J. Olivier and C. M. Sorensen, Phys. Rev. A 41, 2093 (1990).

[12] J. P. Wilcoxon, J. E. Martin, and D. W. Schaefer, Phys. Rev. A 39, 2675 (1989).

[13] B. J. Olivier and C. M. Sorensen, J. Colloid Interface Sci. 134, 139 (1990).

[14] J. E. Martin, Phys. Rev. A 36, 3415 (1987).

[15] A. Chatterjee, M. Kerker and D. D. Cooke, J. Colloid Interface Sci. 53, 71 (1975).

[16] S. E. Devir (Weinstock), J. Colloid Interface Sci. 18, 744 (1963).

[17] G. Nicolaon, M. Kerker, D. D. Cooke, and E. Matijevic, J. Colloid Interface Sci. 38, 460 (1972).
[18] S.-N. Shon, G. Kasper, and D. T. Shaw, J. Colloid Interface Sci. 73, 233 (1980).

[19] P. E. Wagner and M. Kerker, J. Chem. Phys. 66, 638 (1977).

[20] M. von Smoluchowski, Phys. Z. 17, 585 (1916).

[21] M. von Smoluchowski, Z. Phys. Chem. 92, 129 (1917).

[22] M. L. Brodie, Ph.D. thesis, Massachusetts Institute of Technology, 1988 (unpublished).

[23] T. W. Taylor and C. M. Sorensen, Phys. Rev. A 36, 5415 (1987).

[24] N. A. Fuchs, Mechanics of Aerosols (Pergamon, New York, 1964).

[25] C. N. Davies, Proc. Phys. Soc. (London) 15, 61 (1943).

[26] K. W. Lee, J. Colloid Interface Sci. 92, 315 (1983).

[27] C. M. Sorensen and T. W. Taylor, Phys. Rev. A 33, 1411 (1986).

[28] B. J. Berne and R. Pecora, Dynamic Light Scattering (Wiley, New York, 1976).

[29] D. E. Koppal, J. Chem. Phys. 57, 4814 (1972).

[30] E. Moreels, W. DeCeuninck, and R. Finsy, J. Chem. Phys. 86, 618 (1987).

[31] D. J. Coumou, J. Colloid Interface Sci. 15, 408 (1960).

[32] D. J. Coumou, E. L. Mackor, and J. Hijmans, Trans. Faraday Soc. 60, 1539 (1964).

[33] D. J. Coumou and E. L. Mackor, Trans. Faraday Soc. 60, 1726 (1964).

[34] M. Kerker, The Scattering of Light and Other Electromagnetic Radiation (Academic, New York, 1969).

[35] H. Denman, W. Heller, and W. Pangonis, Angular Scattering Functions for Spheres (Wayne State University Press, Detroit, 1963). 